Cahiers d'études africaines

\title{
Un dimanche à Kissidougou
}

L'humanitaire et l'Afrique du postcolonial au global

Sunday in Kissidougou. Humanitarian Action and Africa, from Postcolonial to Global

\section{Michel Agier}

\section{OpenEdition}

\section{Journals}

Édition électronique

URL : https://journals.openedition.org/etudesafricaines/16441

DOI : 10.4000/etudesafricaines. 16441

ISSN : $1777-5353$

\section{Éditeur}

Éditions de l'EHESS

\section{Édition imprimée}

Date de publication : 20 novembre 2010

Pagination : $981-1001$

ISBN : 978-2-7132-2252-8

ISSN : 0008-0055

Référence électronique

Michel Agier, «Un dimanche à Kissidougou », Cahiers d'études africaines [En ligne], 198-199-200 | 2010, mis en ligne le 02 janvier 2013, consulté le 22 avril 2022. URL : http://journals.openedition.org/ etudesafricaines/16441; DOI : https://doi.org/10.4000/etudesafricaines.16441

Ce document a été généré automatiquement le 22 avril 2022

(c) Cahiers d'Études africaines 


\title{
Un dimanche à Kissidougou
}

\author{
L'humanitaire et l'Afrique du postcolonial au global \\ Sunday in Kissidougou. Humanitarian Action and Africa, from Postcolonial to \\ Global
}

Michel Agier

1 Kissidougou est une ville de la Guinée forestière d'environ 70000 habitants près de laquelle étaient implantés en 2003 trois camps de réfugiés : Boreah, Kountaya, Telikoro, soit un peu plus de 32000 réfugiés, libériens et sierra-léonais. Les effets démographiques du conflit de la Mano River sur cette région de Guinée entre 1989 et 2004 ont été importants, fluctuants et difficiles à saisir. Quelques années plus tôt, en 1999, l'ensemble de la région de la Guinée forestière aurait compté un peu plus de 400000 réfugiés (chiffres redescendus à 178000 en 2001) pour une population locale d'environ 900000 habitants (Cirad 2001; Imbernon 2008). C'est à Kissidougou que se trouvaient les bureaux et les résidences des oNG qui intervenaient dans les camps environnants.

2 Un dimanche après-midi de septembre 2003 à Kissidougou, quelques travailleurs expatriés se retrouvent «chez Enfants réfugiés du Monde » pour jouer une partie de volley-ball. Six contre six : deux ERM, trois Croix-Rouge internationale et une HCR d'un côté ; deux MSF, deux ACF, un HCR et un PU de l'autre ${ }^{1}$. Les mêmes personnes, avec quelques autres, se retrouvent aussi le samedi soir pour célébrer un départ, une arrivée, ou pour une "fête à thème »: on se retrouve "chez ACF ", «chez MSF » ou «chez Première urgence ». Ces rencontres, ces relations vite tissées, forment une communauté expatriée importante dans la petite ville de Kissidougou. Deux restaurants de cuisine internationale et quelques magasins alimentaires tirent un bénéfice de leurs consommations de type européen. Le maire de la ville, quant à lui, apprécie la présence des ONG qui apportent, pour la plupart, une aide aux habitants de la préfecture de Kissidougou, en plus de l'aide, majeure, aux réfugiés.

3 Les travailleurs expatriés des ONG sont des volontaires plutôt jeunes, la tranche d'âges des 25-35 ans est de loin la plus représentée, ils sont européens, américains ou australiens, et leurs motivations sont diverses : compassion religieuse, engagement 
social envers les plus faibles, désir d'une vie de baroudeur, engagement politique altermondialiste. Ils passent la semaine à régler les problèmes concrets liés à la réalisation de leur mission humanitaire (problèmes logistiques, gestion du personnel, organisation de réunions de « sensibilisation »). L'action au sein de camps relativement stabilisés a quelque chose de répétitif, d'un peu routinier, et cela déroute certains des volontaires qui s'attendaient à des expériences pleines d'aventures. Celles-ci arrivent parfois, mais dans d'autres cadres, plus rares, comme les tournées dans les centres de réception à la frontière.

4 Le dimanche, chacun essaie aussi, dans la mesure du possible, de tisser des relations personnelles plus étroites avec des Guinéens collaborant avec les onG. Certains vont déjeuner chez l'un ou l'autre et faire connaissance avec leur conjoint et leurs enfants. D'aucuns, parfois, sont invités au baptême de l'enfant d'un employé de l'ong ou passent l'après-midi dans la cour d'un autre, quelques-uns se retrouvent avec les habitants de Kissidougou à la messe de l'église catholique (la ville compte à peu près autant de catholiques que de musulmans).

5 Tous, cependant, savent bien qu'ils sont là pour peu de temps. Trois mois, six mois, jusqu'à un an, le turnover est important, c'est le principe des ONG, soit parce que les volontaires vont reprendre leurs activités dans leur propre pays après cette parenthèse humanitaire, soit parce que les organisations considèrent que les conditions de vie et de travail sont difficiles et que les missions doivent être de courte durée. Aussi attirés soient-ils par la rencontre " avec les gens ", ils restent marqués par la fonction qui les a amenés là, une fonction d'assistance par essence non relationnelle mais "biopolitique ", c'est-à-dire un traitement des autres en tant que victimes et aussi longtemps qu'ils sont considérés sous ce point de vue qui n'implique ni singularités, ni subjectivité, ni relations. C'est parce que ces autres sont "victimes» que les humanitaires sont là, en séjour à Kissidougou, même si chacun donne à sa présence un sens moral, politique ou culturel en plus.

\section{Questions d'actualité postcoloniale}

6 Dans ce pays, qui a fait partie de la colonie française jusqu'en 1958, comme en Afrique d'une manière plus générale, le déploiement humanitaire des années 1980, 1990 et 2000 , représente une forme de la présence européenne correspondant en grande partie à ce qu'on peut appeler une "actualité postcoloniale ». Le thème, on le sait, porte à discussion (Smouts 2007)2 ${ }^{2}$. Je souhaite l'aborder, pour ma part, à partir de la manière dont il s'incarne effectivement sur le terrain, la prise en compte de son historicité impliquant qu'on s'inquiète de sa présence, au double sens du préfixe " post- », à la fois passé révolu, mais toujours référencé et ainsi actuel. Il s'agit de sortir de la polémique académique à propos de la légitimité et de l'utilité, ou non, d'une ligne de recherche "études postcoloniales ", d'en sortir par l'enquête, sans nier pour autant les questions et les contextes qui ont fondé cette polémique. Ce sera l'objet d'un détour, dans les lignes qui suivent, où j'aborderai la question d'un point de vue politique d'abord, puis méthodologique.

7 La polémique postcoloniale s'inscrit pour une part dans des relations épistémologiques "franco-américaines" plus que cinquantenaires. Ces échanges ont une histoire d'allers- retours et de "miroirs" dont les principales étapes se situent dans des contextes politiques et idéologiques très marquants: depuis l'exil américain des 
intellectuels français et plus largement européens pendant la Seconde Guerre mondiale et les liens forts maintenus à leur retour après-guerre, jusqu'aux débats politiques et philosophiques à portée universelle menés dans la France de la décolonisation africaine et de la critique post-soixante-huitarde, dont les mots résonnent encore aujourd'hui bien loin du périmètre européen, et enfin jusqu'aux tentatives actuelles de penser le monde de l'après guerre froide dans sa globalité et sa multipolarité (L'Homme 2008), on constate que la circulation et les échanges intellectuels se sont à peu près toujours déroulés au sein de contextes politiques nationaux et internationaux en tension et en conflit. Pas de tour d'ivoire, pas de pure théorie, les enjeux politiques nationaux, régionaux puis, de plus en plus, mondiaux transparaissent à chaque intervention « pour » ou « contre » la question postcoloniale.

8 Pour une autre part donc, la polémique postcoloniale se développe bien au-delà du face-à-face culturel franco-américain qu'il serait réducteur et dérisoire de convoquer encore en 2010. Elle s'inscrit bien plus largement dans la recherche actuelle, aussi inquiète que légitime, de la meilleure manière d'entrer dans la mondialisation intellectuelle, au double sens où chacun veut légitimement y tenir une place - pour aller, disons, vers une multipolarité intellectuelle -, et où nous sommes tous à la recherche d'une intelligence du monde en train de se faire. «Faire monde » ou « entrer dans le monde ", le ressentir et l'expérimenter comme monde commun et sensible à cette échelle-là, savoir en dire les hiérarchies et les inégalités, est devenu une problématique partagée après la fin des mondes politiques coloniaux, ce qui pose assez logiquement, sur les terrains contemporains de l'anthropologie comme sur ceux de l'étude des biographies individuelles ou des histoires collectives, la question de savoir dire ce qui s'est perdu et ce qui reste de ces mondes ${ }^{3}$.

9 Si l'on veut revenir au terrain donc, et saisir les situations observées à la fois dans leur totalité et leur historicité, il convient de sortir de la polémique postcoloniale en dépassant aussi les orgueils et purs mouvements d'humeur que cette polémique révèle face au défi commun de réussir à penser le mouvement du monde. Les dépasser en les mentionnant à leur place et dans leur « moment » cependant. D'un côté les humeurs de la "french theory" d'aujourd'hui qui aurait « déjà donné » face à des subalternistes qui réactualiseraient les identités les plus figées ${ }^{4}$. De l'autre, les rancœurs toujours présentes d'intellectuels qui restent ancrés sur la même "marge » que les régions d'où ou dont ils parlent, se défendant pourtant d'être revanchards ${ }^{5}$. Il est vrai que leur attachement à la pensée critique de l'Europe, et de la France en particulier, n'a pas trouvé, pour diverses raisons politiques et institutionnelles, l'hospitalité et les échanges souhaités en retour, et que c'est bien aux États-Unis, plus riches, efficaces et ouverts, que les chercheurs en histoire et en sciences sociales et politiques d'Afrique noire, d'Inde et d'Amérique latine ont trouvé leur place d'une manière aussi « naturelle » que structurelle. Le ton irrité tend d'ailleurs à corroborer une des clés de la polémique, à savoir que le courant dit des « études postcoloniales » incarne à l'excès, dans le langage académique, la revendication politique d'un partage du pouvoir à l'échelle mondiale, qui ne peut qu'irriter en retour les universitaires et chercheurs français qui, au-delà d'un «narcissisme politique, culturel et intellectuel» (Mbembe 2005b: V) bien mis à mal, voient dans le même temps leur propre monde de la recherche devenir, de réforme en réforme ministérielle, plus étroit et précaire, peu accueillant en fait et, malgré les bonnes intentions affichées, en somme plus « provincial » et peu disponible pour se projeter dans un avenir mondialisé. La pratique et les discours académiques semblent tourner en sens inverse, ce qui est effectivement irritant, mais renvoie plutôt 
à la nécessité d'un combat politique « interne ", français et européen, qu'à des combats idéologiques « externes ».

La polémique sur le bien-fondé ou non d'une ligne de recherches postcoloniales relève ainsi de positionnements autant politiques et institutionnels qu'épistémologiques ou théoriques ${ }^{6}$. Mais elle laisse à d'autres le soin de s'interroger pour de vrai sur l'historicité (post)coloniale, autrement dit et pour être concret, sur cette part postcoloniale de la généalogie du contemporain. Après l'ère du "développement", celle de l'humanitaire offre en Afrique un terrain privilégié à l'étude de cette question, comme on le verra plus loin, après un dernier point de méthode.

11 Il convient en effet de reprendre le fil de l'analyse de situation, poursuivre l'enquête du point de vue de l'historicité et de l'ethnographie politique des situations dans leur contexte. Après les analyses de la "situation coloniale " (Balandier 1992), poursuivre cette anthropologie des situations sans préjuger de la définition du contexte, affirmant plutôt son énigme et son instabilité. On notera en passant que les commentaires de la " situation coloniale » oublient généralement la relation directe et contemporaine de ce concept avec l'anthropologie des situations, inaugurée au même moment au Rhodes Livingstone Institute de Lusaka, notamment dans les enquêtes majeures de Max Gluckman (1940) ${ }^{7}$ et Clyde Mitchell (1956). Analyser la situation dans sa totalité, dans ses multiples contraintes et dans sa formation processuelle, comme le propose alors Balandier, trouve écho dans ce courant marquant dans les années 1950, même s'il est minoritaire, qui va progressivement constituer l'analyse situationnelle, et déjà poststructuraliste, non pas selon le point de vue holiste et externaliste du «fait social total » qui suppose l'existence d'une "société » déjà là et juste incarnée et individuée, mais selon une démarche interne et inductive: l'immanence du contexte dans la situation est à rechercher dans l'enquête sur chaque configuration; ce qui revient à poser, en toute rigueur, l'indéfinition du contexte a priori. Autrement dit, et pour le dire avec les mots clairs de Jean Bazin (1996), «la société n'est pas une chose que je peux observer. Si éloignée ou petite soit-elle, le point de vue de Sirius ne m'est pas davantage accessible. Je n'observe jamais que des situations $»^{8}$. Si je rappelle ce point de méthode, c'est que la question n'est pas de nommer, sous forme éventuellement propagandiste, le tout d'une "société", coloniale puis postcoloniale ou non, mais de reconnaître comment et dans quelle mesure ces déterminations agissent sur la définition du monde sensible tel que peut l'appréhender l'analyse situationnelle9. Question de méthode ou de regard, certes, mais qui relève bien d'un choix politique.

Si l'on peut dire, et à juste titre, que Gérard Althabe a été le premier anthropologue à mener des études « subalternes » et " postcoloniales ", à l'époque charnière d'après les Indépendances, encore convient-il de préciser la position épistémologique originale qui lui permet de tenir cette position théorique, plus souvent définie d'ailleurs comme une "sociologie de la décolonisation": c'est celle d'une ethnographie politique des situations, qui décrit de l'intérieur et avec toute la minutie nécessaire et possible de qui se place et circule à l'intérieur de la situation, comment la forme de domination coloniale «s'actualise » et travaille dans les figures imaginaires blanches et noires $\mathrm{du}$ culte du tromba à Madagascar dans les années 1960, ou quelques années plus tard dans l'organisation politique et symbolique des ségrégations urbaines à Kinshasa (Althabe $1997,2002)^{10}$.

13 C'est avec cette distance autant théorique que méthodologique à l'égard de la polémique postcoloniale, mais tout en gardant, pour la mettre à l'épreuve, la question 
de la continuité autant que des ruptures postcoloniales, que je reviens à l'analyse du contemporain de l'Afrique, qu'il convient ici de distinguer, en toute rigueur, de ce qui reste des pratiques et savoirs coloniaux dans les ex-métropoles européennes. C'est l'Afrique elle-même qui est le terrain d'une problématique "pragmatique» de la "postcolonie " selon le mot d'Achille Mbembe (2005b:40), un terrain où se pose de manière bien distincte la question de la domination postcoloniale et de la continuité dans ce cadre présent, d'un régime de violence postcolonial inscrit dans des représentations et manières de faire comme dans des pratiques, des institutions et des espaces.

\section{Le mouvement humanitaire occidental : ruptures et continuités post coloniales}

Dans ce contexte, l'humanitaire occupe, au même titre, par exemple, que le tourisme, les migrations ou l'Internet, une présence forte, pérenne et donc une capacité transformatrice à venir, dont la problématique doit inclure son historicité. En quelle mesure, dans quel contexte et avec quelle intensité l'intervention humanitaire définitelle une situation dont on peut dire qu'elle relève, au moins en partie, d'une actualité postcoloniale ? L'humanitaire dans son ensemble apparait d'abord, aujourd'hui, comme une des composantes essentielles de la mondialisation en cours - il convient d'en retracer les grandes étapes, j'y reviendrai plus loin. Mais une part de la généalogie de cette fonction mondialisante de l'humanitaire, en particulier du point de vue des relations des savoirs gestionnaires ou "biopolitiques", s'est accumulée au long de l'expérience historique des empires coloniaux et relève bien d'une généalogie postcoloniale. Une autre part semble s'être construite contre elle, ce qui est une autre manière de se placer dans la filiation.

En effet, si l'on parcourt cette généalogie sur les terrains humanitaires, on observe que la relation Blancs-Noirs qui s'y joue aujourd'hui, particulièrement en Afrique de l'Ouest et centrale, s'est construite politiquement et, pourrait-on dire, culturellement en rupture avec celle qui s'était nouée entre, d'une part une France d'abord impériale et coloniale puis «coopérante» et «developpementiste» et, d'autre part, une Afrique engagée de diverses manières dans des relations étroites, notamment politiques, avec une partie au moins des acteurs du pays (ex)colonisateur. En ce temps-là l'imbrication était forte, non seulement sur le terreau des célèbres affaires "françafricaines " postcoloniales (et qui, elles, perdurent dans le soutien français à plusieurs dirigeants africains maintenus au pouvoir en bonne partie grâce à ce soutien), mais aussi sur le terrain d'un engagement et d'un échange politiques, plus anciens et très largement perdus aujourd'hui, autour des mouvements politiques, syndicaux ou intellectuels de la décolonisation et des Indépendances africaines ${ }^{11}$. Car, si l'époque coloniale fut marquée par des spoliations importantes de ressources naturelles, par des formes extrêmes d'exploitation de la force de travail colonisée, par des violences politiques et des brutalités raciales idéologiques et physiques, l'imbrication et la proximité contenaient également une relation politique et sociale qui joua un rôle inclusif et favorisa, dans certains domaines au moins, une appropriation africaine de la France tout comme une africanisation de la France.

16 Cette domination complexe et contradictoire a laissé plus que des traces, un lien; ce lien a perduré après les Indépendances tout en se transformant. Un sentiment 
d'appartenance à une histoire commune, une formation culturelle qui inclut ce qui venait des pays colonisateurs (langue, musiques, idéologies politiques), ont ainsi marqué plusieurs générations d'Africains avant et après les Indépendances ${ }^{12}$. L'Empire avait été cette unité englobante au sein de laquelle s'étaient développés différents types de sociabilités : violentes, autoritaires, paternalistes... ou solidaires. Les prolongements sociaux et économiques de cette relation "interne", c'est-à-dire tissée au sein de l'Empire colonial, ont laissé après les Indépendances - dans les phases de la «coopération » et du " développement » - plus de frustrations que de réussites.

Ces échecs et, en d'autres mots, ces leurres, de même que les désengagements qui ont suivi la fin de la guerre froide, en particulier sur le plan économique (les « ajustements structurels » des années 1990, le passage à l'Euro et le détachement du franc CFA, etc.), ont été perçus par nombre d'Africains comme un abandon brutal, comparable sinon égal aux spoliations et dominations passées. Cette violence de l'attitude des pays (ex)colonisateurs, consistant à ne pas maintenir pour l'intégration au monde- semondialisant l'engagement qui avait été réalisé pour la spoliation coloniale, n'a pu qu'entretenir nostalgies, ressentiments et finalement aboutir au distancement d'aujourd'hui, non sans regrets. Une part de la mondialisation africaine échappe ainsi, de fait, au cadre postcolonial, quand une autre part se construit en conflit avec lui. Dans le cadre des politiques migratoires restrictives que les gouvernements européens ont engagées à la fin des années 1990 en même temps qu'ils redéfinissaient les frontières de l'Europe à 25 puis 27, les migrants qui viennent du sud de la Méditerranée et qui sont confinés dans des espaces et statuts liminaires se voient exclus de fait du cadre postcolonial de la mondialisation lui-même: soit ils se retrouvent "sans papiers", " clandestins ", et ainsi rejetés au-delà même de la reconnaissance subalterne coloniale, soit ils orientent eux-mêmes leurs trajets ailleurs (Bazenguissa-Ganga \& Mbembe 2008 ; Mabanckou 2009 ; Migreurop 2009).

18 L'engagement humanitaire en Afrique s'est d'abord inscrit dans ce contexte, il a été le substitut d'une domination domestique voire intime dans la colonie et la postcolonie, auxquelles il a semblé d'abord s'opposer politiquement, moralement et d'une certaine façon, cette rupture a semblé fonder la culture de cet engagement à ce moment-là. L'humanitaire a ainsi connu une étrange heure de gloire africaine dans les années 1980 et surtout 1990. Sa montée en puissance fut contemporaine de cet abandon des anciens empires coloniaux et une de ses conséquences logiques. Conséquence pour les individus qui s'y engagèrent et voulurent apporter dans l'urgence une réponse à ses effets désastreux, quitte à avoir toujours le sentiment d'être une présence aux effets dérisoires face à la tâche salvatrice assignée, et tirant de ces échecs personnels une rancœur parfois ambiguë et sans issue, dirigée contre l'incompréhension ou les traditions des «bénéficiaires" autant que contre l'aveuglement des commanditaires. Conséquence aussi pour le sens global de l'intervention humanitaire en Afrique foncièrement associée à la continuité de la présence blanche et à la dépolitisation des relations entre le premier monde (blanc) et l'Afrique. Car la rhétorique d'une rupture culturelle et morale s'est construite sur le fond d'une continuité sociale, même transformée. Le lien colonial, à la fois proche et dominateur (paternaliste) d'une part, solidaire ou conflictuel (politique) d'autre part, semblait avoir été rompu; mais le rapport de domination est ce qui seul est resté dans la continuité postcoloniale... Ainsi, localement, les humanitaires ont simplement pris le relais des colons et des fonctionnaires de la colonie d'abord, des coopérants et des développeurs ensuite, pour incarner la nouvelle modalité de présence et la nouvelle domination des Blancs. Au 
cœur des affects et des idéologies des uns et des autres, il y a dans l'intervention humanitaire autant de la continuité que de la rupture avec l'intervention coloniale. C'est un reste, ou une "ruine " autant culturelle que matérielle d'un empire disparu (Stoler 2008), ruine encore vivante et en partie active, d'une certaine façon, sur laquelle se tissent de nouvelles relations et de nouvelles tentatives de donner du sens à la présence de ces « nouveaux » Blancs, en affrontant et négociant avec d'autres langages, notamment celui de la puissance occidentale associée à la compassion, ou celui de l'image victimaire d'une Afrique qui restera là, gisant sans projet dans un éternel chaos. Et il n'est pas sûr que les deux principales caractéristiques de l'intervention humanitaire, à savoir la situation d'exception qu'institue l'urgence, et l'extraterritorialité du camp, soit absolument nouvelles pour l'histoire locale.

Pour corroborer ce commentaire, revenons une seconde fois sur le terrain guinéen, pour faire le double constat d'un défaut de communication, non par essence (ce serait faire injure à l'intention des travailleurs humanitaires que de le prétendre si vite) mais par absence de nécessité. Comme si la vertu de l'attention à l'autre s'épuisait d'ellemême là où sa nécessité manque.

\section{L'intervention humanitaire comme situation postcoloniale dans un paysage global}

20 À Kissidougou ce dimanche de septembre 2003, jour de repos des humanitaires, même s'ils le regrettent ils ne parviendront pas à parler la langue locale, parce qu'ils n'auront pas eu le temps de l'apprendre, et surtout parce que ce n'est pas utile à la réalisation de leur mission. Ils ne connaîtront pas « les gens » parce qu'on ne leur demande pas cela non plus. Les dimanches vont s'étirer, ils vont finir par préférer l'entre-soi éphémère des « fêtes » du samedi soir et des parties de volley-ball du dimanche.

Puis le lundi matin, le cortège des $4 \times 4$ blancs marqués de leurs logos respectifs va les reconduire vers le quotidien des camps. C'est là que la relation humanitaire, tout à la fois postcoloniale et mondialisée, va se construire de manière plus radicale, plus dure, en se mettant en scène sur son propre terrain, celui où, de manière totale voire totalitaire, l'ordre et la pensée humanitaires sont omniprésents et semblent « définir la situation ».

Parmi les trois camps proches de Kissidougou, celui de Boreah est situé à une trentaine de kilomètres et un peu plus d'une heure de piste de la ville. Il abrite 7500 réfugiés en septembre 2003, et au moins douze organisations internationales: le Programme alimentaire mondial de l'ONU (PAM) fournit et stocke des produits alimentaires ; le HCR coordonne l'ensemble des interventions extérieures dans le camp; International Rescue Comittee (IRC, États-Unis) s'occupe d'écoles primaires ; Médecins sans frontières (MSF section belge) gère les deux postes de santé du camp; Enfants réfugiés du Monde (ERM, France) mène des activités de loisirs, de récréation et de dialogue en direction des enfants; Action contre la faim (ACF, France) est présente avec des projets agricoles; Première urgence (PU, France) s'occupe de la distribution de nourriture et d'un élevage de poulets; la Croix-Rouge guinéenne intervient dans le management du camp pour le " volet social » et distribue des bâches plastifiées pour faire le toit des cases; American Refugee Comittee (ARC, États-Unis) propose des prêts d'argent pour soutenir des "projets économiques» et intervient également à propos de la violence sexuelle (programme dit GBV : «Gender based violence »); Center for Victims of Torture (CVT, 
États-Unis) pour les soins de santé mentale ; Action of Churches Together (ACT, ÉtatsUnis) propose l'intervention de médecins psychiatres ; l'agence publique allemande de coopération technique (GTZ) intervient pour la construction du camp et l'animation d'un centre de formation professionnelle; on compte également les ONG Save the Children Found (sc-UK, Grande-Bretagne) et le Centre canadien d'études et coopération internationale (CECI, Canada), ainsi qu'une ONG guinéenne pour l'assistance alimentaire aux vulnérables (REPC). Le Relief Activities of Detraumatisation and Improvement of Refugees (RADIR) est une oNG créée sur place par quelques réfugiés qui cherchent à développer des activités sportives, théâtrales, des jeux de rôles, etc. Le Bureau de coordination des réfugiés (BCR, représentant le gouvernement guinéen) a un local et contrôle une "brigade mobile de sécurité » (BMS, de la police guinéenne), ainsi que quarante-trois "assistants de sécurité " sélectionnés parmi les réfugiés. Enfin, un " comité des réfugiés » a été mis en place sous le contrôle du HCR et du BCR.

Cet excès d'encadrement laisse perplexe. C'est une présence très visible, mais moins efficace que ce que peuvent laisser penser la lecture des rapports hebdomadaires ou mensuels des ONG, ou le nombre incalculable de réunions de coordination «interagencies", de réunions "de sensibilisation", de grands panneaux et de drapeaux des organisations onusiennes et des ONG qui décorent le paysage très " global » du camp, ou encore le manège des $4 \times 4$ blancs qui circulent dans son enceinte. Pour les réfugiés, cette présence forme seulement une partie du contexte de leur vie, celle qui les considère comme des victimes, comme les ayants droit à une assistance qu'ils ne contrôlent pas et en face de laquelle ils ne peuvent être que spectateurs, quémandeurs, plaintifs - dans le meilleur des cas, roublards, "tricheurs", "manipulateurs » ou "profiteurs du système », pour employer quelques-uns des nombreux termes qui les qualifient en tant que réfugiés.

L'injonction urgentiste impose en outre aux travailleurs des ONG internationales d'avoir toujours quelque chose d'urgent à faire ; un empressement qui contraste avec la lenteur caractérisant le rythme des réfugiés dans les camps - cette lenteur étant, elle, la forme visible de plusieurs états possibles : souffrance, ennui, ou mélancolie attachée au lieu perdu et à l'incertitude du devenir, tissage de nouveaux liens sur place, transformation des habitations. Dans le présent suspendu de la vie des camps, se croisent ainsi les humanitaires expatriés qui s'affairent dans une urgence sans fin, et les réfugiés qui essaient de se repérer et de se refaire une vie dans ces nouveaux lieux et dans la lenteur ${ }^{13}$.

Mais cette coprésence dissonante entre le dispositif humanitaire et les réfugiés, n'est qu'un aspect du malentendu inhérent à toute situation humanitaire, qui est en partie, mais en partie seulement, postcoloniale, à l'image du processus de mondialisation dont l'humanitaire est l'une des composantes.

\section{Les trois âges de l'humanitaire. La fabrication d'un récit mondial}

Un long et lent processus a caractérisé le développement, la justification et l'institutionnalisation de l'humanitaire dans les pays européens ${ }^{14}$. Mais une accélération et une mondialisation rapide de cette histoire ont marqué les quatre dernières décennies. Cette histoire récente a constitué le récit humanitaire comme un des grands récits de la mondialisation, au sens où son action est une de celles qui constituent le monde en tant que monde. Cette part humanitaire à l'édification du 
monde mondialisé se fonde autant sur les réseaux mondialisés d'un gouvernement humanitaire des indésirables, que sur la référence nécessairement consensuelle à une humanité conçue comme identité dont le double n'est pas un «autre » égal, mais la victime absolue - un humain diminué, altéré et finalement imparfait (Agier 2010a) ${ }^{15}$. Les coordinations internationales autant que locales des interventions humanitaires (les clusters), leurs liens (parfois involontaires et forcés, parfois plus conscients, stratégiques) avec le dispositif des interventions militaires à l'échelle mondiale, notamment onusienne, ou encore la formation d'un paysage mondial de camps sous leurs multiples formes et fonctions (ibid. 2010b), sont quelques-uns des aspects de cette part humanitaire de la mondialisation, autant gouvernementale qu'idéologique. Comme les autres cependant, le récit humanitaire mondial n'en est qu'à son commencement et c'est en grande partie en Afrique que se joue son avenir. Avant d'y venir, on commentera très sommairement les trois étapes les plus marquantes de son histoire récente, sur fond d'une succession de crises.

Premier temps, celui des French doctors. C'est le temps des engagements militants des années 1970 où l'on voit naître dans le «Premier monde » des mouvements qui se sont développés dans la contestation à la fois anti-colonialiste en soutien aux mouvements de libération nationale du Sud, et postmoderniste au sein des sociétés du Nord, préparant les idéologies politiques et morales en vigueur au tournant du $\mathrm{xxI}^{\mathrm{e}}$ siècle ainsi que leurs débats intellectuels: le mouvement humanitaire contemporain, l'écologie comme mode de vie et comme politique, les cultures urbaines alternatives, l'ethnopolitique... Ils sont porteurs d'une critique généralisée contre le système de production et de consommation occidental, contre ses valeurs morales et politiques, et à la recherche de ruptures et d'alternatives. Pour l'humanitaire, la guerre du Biafra d'une part, les salles de rédaction de l'hebdomadaire médical français Tonus d'autre part, servent de théâtre à la création de Médecins sans frontières en 1971 (Brauman 2006) ${ }^{16}$.

Deuxième temps, deuxième configuration: le tournant des années 1980-1990 correspond à la réorganisation politique et militaire du monde avant et après la chute du mur de Berlin. C'est la grande époque des opérations humanitaires très médiatisées, portant loin l'œil et la main du «Premier monde». C'est l'époque de l'internationalisation des ONG et de leur professionnalisation, des rapprochements entre oNG et agences onusiennes. La création d' $\mathrm{OCHA}^{17}$ au sein de l'oNU (Organisation des Nations Unies) en 1992 a constitué une tentative visant à coordonner l'action humanitaire. Des conflits de compétence sont apparus, notamment sur les terrains africains, entre le HCR et l'OCHA, à propos de la gestion des "déplacés internes ", dont le nombre est aujourd'hui évalué à vingt-cinq millions dans le monde, dont douze millions en Afrique. Au niveau européen, $\mathrm{ECHO}^{18}$ a été créé en 1992, et occupe une place centrale dans le financement et donc dans le pilotage de l'intervention des ONG des pays européens, en particulier de la vaste nébuleuse des petites oNG sans indépendance financière. Le dispositif se compose également de très grandes ong d'ampleur internationale. Les unes sont issues d'organisations nationales apparues au cours de la première moitié $d u x^{e}$ siècle, les autres sont nées dans les années 1970. Cependant, pour toutes ces organisations, les années 1980-1990 ont été décisives du point de vue de leur organisation à l'échelle planétaire. Ce fut notamment le cas pour le réseau international CARE (États-Unis), pour IRC (États-Unis), OXFAM International (1995), pour l'Alliance internationale Save the Children (1997) ou le mouvement MSF. 
29 Enfin, troisième temps : le contexte de la "guerre contre le terrorisme ", après le 11 septembre 2001, ressemble étrangement à une science-fiction, pourtant il prolonge et hâte la mise en œuvre effective et à l'échelle mondiale d'une police d'empire... et l'humanitaire est sa "main gauche »: le gouvernement humanitaire qui se forme aujourd'hui est en train de devenir un dispositif performant et mondialisé, composé d'experts et d'expatriés se donnant pour tâche d'assurer la gestion du chaos, le contrôle et le confinement des effets "catastrophiques» de la partition du monde entre les zones de prospérité et les surnuméraires. Cela structure aussi un marché, où se diffuse rapidement l'idée que l'affaire devient trop sérieuse pour la laisser aux seules mains des ONG. Un caractère plus «entrepreneurial » que «militant " se développe dans la culture professionnelle des très grandes ONG (Abélès 2006; Agier 2008: 295-312), en même temps que la privatisation de l'intervention humanitaire et, plus encore, des opérations de contrôle et sécurité sur les mêmes terrains (Klein 2008).

À la suite du tremblement de terre de Haïti en janvier 2010 et après la concurrence désordonnée et les conflits parmi les agences onusiennes, l'armée américaine, et les ONG internationales lors de la mise en œuvre de l'intervention internationale, une exministre française de l'uMP ${ }^{19}$ a lancé le projet d'une «force humanitaire mondialement coordonnée ", les « casques rouges ", projet qui ne sonne étrange ou farfelu que si l'on occulte le fait que ces vingt dernières années ont été déjà marquées par une mondialisation de la gouvernance des très grandes onG humanitaires. Sans attendre ces hypothétiques « casques rouges ", le chef d'état-major de l'отAN ${ }^{20}$ a appelé, de son côté, au début de l'année 2010, à une réorientation stratégique de l'OTAN en Afghanistan: pour ce faire, il a proposé de remplacer le « hard power » de l'armée, mal accepté par les Afghans, par le «soft power» des ONG humanitaires ${ }^{21}$.

31 En un mot, le moment est difficile, fatal même, pour un " mouvement » humanitaire que beaucoup ont vécu comme une forme alternative, concrète, effective, de solidarité internationale, en allant sur le terrain à la rencontre des "autres" lointains, vulnérables et sans ressources. C'est cette crise qui fait débat en 2010 dans les milieux intellectuels de l'humanitaire, même si le diagnostic et les solutions ne sont pas partagés par tout le monde ${ }^{22}$.

\section{Images africaines de l'humain selon l'humanitaire}

32 Le signe et la condition de la puissance de l'humanitaire résident dans l'étrange présence-absence de ses ayants droit. Symboliquement omni présent mais intellectuellement et politiquement absent, ce monde à part - le monde, inévitablement ambigu, des "victimes" - est bien parfois exhibé sous des formes outrées : celle du journal télévisé montrant fugitivement des images de désespoir ou celle de l'affiche de telle organisation humanitaire avec gros plan sur les yeux suppliants et le corps squelettique d'un enfant noir et nu... Mais il n'a aucune place dans les conceptions du monde que le monde occidental produit. Que dire de ces ayants droit, sinon les plaindre ou les condamner? Une forme de relégation intellectuelle accompagne la mise à l'écart des populations "victimes » et tout autant indésirables, lesquelles n'apparaissent en général que sous la forme révélée de "l'impensable », de "l'intolérable», de "l'indicible». Autant de figures extrêmes de l'altérité et de l'exotique contemporains qui provoquent la sidération et confirment ainsi, par une mise en scène émotionnelle, le caractère exceptionnel de leur incursion inattendue 
dans les images du monde. Et le geste lié à l'émotion (celui du bienfaiteur qui donne ou du volontaire qui décide de partir) est directement relié à l'image de la souffrance qui est une image "révélée » de forme absolue : sans avant et sans après, sans contexte. Peur, bienfaisance, souffrance, révélation absolue : ces émotions qui donnent l'élan de soi vers le monde composent les ressorts les plus puissants du récit humanitaire. Ce geste qui se pense comme une réponse immédiate à la révélation de l'extrême souffrance peut d'ailleurs être assumé par leurs auteurs comme "spontané", "révolté ", "non réflexif », et finalement tout autant dominateur que donateur. Le geste se veut soignant ou sauveur, mais il construit une victime qui doit garder l'apparence de la victime absolue, sans nom et sans voix: celle-ci doit savoir se conformer à son image, recevoir l'argent sans le "détourner ", sans le "gaspiller ", sans utiliser les aides à d'autres fins que celles conçues par le monde des donateurs (personnes, associations ou pays donateurs), bref sans désordre. La victime doit savoir recevoir en restant à sa place.

Des organisations non gouvernementales ancrées dans le «Premier monde » sont chargées de la fonction morale consistant à transférer le don charitable au loin, et elles garantissent du même coup que tout se passe dans la modalité du spectacle et de la virtualité qui sont des expressions de cette mise à distance (Boltanski 1993; Mesnard $2002)^{23}$. La tranquillité morale de cette compassion déléguée est mise à l'épreuve lorsque des organisations se montrent au grand jour moralement ambiguës, sans que l'ambiguïté fondamentale de l'intervention humanitaire soit elle-même mise en cause, alors qu'elle est à l'origine de son éventuelle violence dans des situations limites. On a ainsi entendu en France, au moment de l'affaire de l'Arche de Zoé à l'automne 2007, que ce serait l'incompétence ou l'immoralité de quelques personnes qui seraient à l'origine du scandale des enfants enlevés/secourus au Tchad pour être adoptés en France. Pourtant, on peut bien, comme l'a fait en particulier Rony Brauman ${ }^{24}$, dénoncer les versions extrêmes et dangereuses de cette métapolitique de l'humain par laquelle certains humanitaires se donnent le droit d'intervenir partout et dans le déni des territorialités politiques (la critique vise «l'irresponsabilité» du fameux «droit d'ingérence » défendu par l'ex-MSF et actuel ministre des Affaires étrangères du gouvernement français, Bernard Kouchner). Il n'empêche que « le ver est dans le fruit » pour le dire crûment, et que, si ces comportements furent possibles, et continuent de l'être, c'est parce qu'il existe aujourd'hui une conception exceptionnaliste de l'Afrique bien ancrée dans la société et les milieux politiques du monde occidental, et profondément compatible avec la situation d'exception instaurée par l'intervention humanitaire dans un espace donné - espace qui se trouve menacé de désocialisation et dépolitisation à chaque nouvelle arrivée de la "souveraineté » humanitaire (Pandolfi 2000). Même les plus critiques et les plus aguerris du mouvement humanitaire n'ignorent pas le fait que l'exception est la règle humanitaire comme elle est celle de la représentation et du gouvernement des indésirables en général, qui combine l'extraterritorialité, l'exceptionnalité, et une forme ou une autre de relégation ${ }^{25}$. L'aile critique du «mouvement humanitaire » reste impuissante face aux effets dépolitisants et finalement déshumanisants de l'imagerie d'une Afrique mineure et victimaire, que l'intervention humanitaire elle-même entretient et dont certains acteurs en son sein ont pu mépriser les institutions politiques, juridiques, d'un État africain, méprisant les personnes elles-mêmes au nom de la représentation humanitaire des victimes sans nom. 
Revenons pour finir, une fois encore vers le camp de Boreah, près de Kissidougou en Guinée, à peu près au même moment que les épisodes décrits plus haut, pour ajouter un élément à la complexité de la situation. En août 2003, des travailleurs d'une ONG humanitaire se font séquestrer par un groupe d'une cinquantaine de femmes réfugiées sierra-léonaises qui les retiennent sous la pluie, dans la « rue » principale du camp, tout un après-midi jusqu'à obtenir des bâches plastifiées pour le toit de leurs cases construites en terre et en chaume, et tout inondées par des pluies diluviennes. Aux travailleurs humanitaires qui ne comprennent pas leur action et leur disent que la livraison des bâches n'est pas du ressort de leur organisation, les femmes répondent que toutes les ONG sont semblables comme tous les réfugiés sont semblables. Après plusieurs heures de confrontation et discussion, elles obtiennent finalement l'engagement par les responsables du camp que leurs bâches plastifiées leur seront livrées dans les jours suivants.

Cette mise en cause de l'ordre normal du camp prend la forme d'un agir politique sur le plus improbable des espaces politiques, le camp de réfugiés incarnant la «scène humanitaire » de la manière la plus aboutie et complète. D'autres conflits du même genre - manifestations devant les compounds humanitaires des camps, boycott de la ration alimentaire du Programme alimentaire mondial, réunions informelles et rédactions de cahiers de doléances, grèves de réfugiés employés dans les camps par les oNG et les agences onusiennes, etc. - ont lieu dans les camps de réfugiés, en particulier dès qu'ils s'installent dans une durée de plusieurs années, dépassant donc de loin le temps de l'urgence. Dans ce cadre, les réfugiés instaurent un face-à-face politique qui les oppose aux « UN » en général ${ }^{26}$, aux Blancs, à la communauté internationale réifiée sous leurs yeux par la présence humaine et matérielle du déploiement humanitaire.

D'autres événements ont eu lieu ces dernières années au Tchad, au Niger ou au Soudan, qui ont vu des enlèvements, assassinats ou expulsions de personnels humanitaires (certes, à chaque fois, par des acteurs bien différents). Ils ne relèvent donc pas du type d'affrontement politique que j'ai pu observer dans divers camps de réfugiés. Cependant, ils désignent l'humanitaire comme ce même "acteur" idéologique d'un conflit, ce qui est apparemment le comble de la politique en situation humanitaire, mais nous renvoie tout autant à l'historicité postcoloniale qu'au devenir mondial de l'intervention humanitaire dans les pays du Sud, et en Afrique en particulier. Une inclusion générale et indistincte des organisations humanitaires, quelles qu'elles soient, dans le wagon de la "communauté internationale ", par des acteurs politiques locaux ou des groupes criminels, en font un humanitaire perçu comme "embarqué ». La violence que cette inclusion autorise à penser, provoque une crise, au sens d'une déstabilisation du sens passé - ce que les travailleurs humanitaires expatriés vivent sur place comme un «malentendu ». Dans les débats qu'on a évoqués plus haut, ce sont cette violence tournée contre l'humanitaire "embarqué » et cette déstabilisation qui amènent certains intellectuels du mouvement humanitaire à appeler de leurs vœux une «dés-occidentalisation» de l'humanitaire. Et le mot blesse, comme il provoque la polémique. On y verra, sans malice, un écho à la polémique postcoloniale dans les sciences sociales, et la nécessité dans les deux cas d'éprouver les effets du décentrement: un rééquilibrage des forces en présence dans la définition et la résolution pragmatique de chaque situation. 


\section{BIBLIOGRAPHIE}

ABÉLÈs, M., 2006 Politique de la survie, Paris, Flammarion.

AGIER, M., 2008 Gérer les indésirables. Des camps de réfugiés au gouvernement humanitaire, Paris, Flammarion («Bibliothèque des savoirs »).

-, 2009 Esquisses d'une anthropologie de la ville. Lieux, situations, mouvements, Louvain-la-Neuve, Academia Bruylant.

,$- 2010 a$ « Humanity as an Identity and its Political Effects. A Note on Camps and Humanitarian Government », Humanity, 1, University of Pennsylvania Press.

-, 2010b « Camps for Foreigners and the Corridor of Exile : A Global Landscape », Transeuropa, 9 : 20-21.

ALTHABE, G., 1997 [1972] Les fleurs du Congo, Paris, L'Harmattan.

-, 2002 [1969] Oppression et libération dans l'imaginaire. Les communautés villageoises de la côte orientale de Madagascar (préf. de G. Balandier), Paris, La Découverte.

AMSELLE, J.-L., 2008 L'Occident décroché. Enquête sur les postcolonialismes, Paris, Stock.

APPADURAI, A., 1996 Modernity at Large. Cultural Dimensions of Globalization, Minneapolis, University of Minnesota Press (éd. française : Après le colonialisme. Les conséquences culturelles de la globalisation, Paris, Payot, 2001).

BALANDIER, G., 1985 [1955] Sociologie des Brazzavilles noires, Paris, Presses de la Fondation nationale des sciences politiques (postface de J. Copans).

-, 1992 [1955] Sociologie actuelle de l'Afrique noire, Paris, PUF.

BAUMAN, Z., 2010 Identité, Paris, L’Herne.

BAYART, J.-F., 2010 Les études postcoloniales. Un carnaval académique, Paris, Karthala.

BAZENGUISSA-GANGA, R. \& MBEMBE, A., 2008 « Mobilités africaines, racisme français... », Vacarme, 43 : 83-85.

BAZIN, J., 1996 « Interpréter ou décrire. Notes critiques sur la connaissance anthropologique », in J. REVEL \& N. WACHTEL (dir.), Une école pour les sciences sociales, Paris, Cerf/EHESS : 401-420.

BOLTANSKI, L., 1993 La Souffrance à distance. Morale humanitaire, médias et politique, Paris, Métailié.

BRAUMAN, R., 2006 Penser dans l'urgence (entretiens avec C. Portevin), Paris, Éditions du Seuil.

CHAKRABARTY, D., 2000 Provincializing Europe. Postcolonial Thought and Historical Difference, Princeton, Princeton University Press.

-, 2009 Provincialiser l'Europe. La pensée postcoloniale et la différence historique, Paris, Éditions Amsterdam.

CIRAD, 2001 « Environnement et populations réfugiés », CIRAD/IRD/HCR/Fond français pour l'Environnement Mondial.

COPANS, J., 2007 « Les frontières africaines de l'anthropologie. Un demi-siècle d'interpellations », Journal des Anthropologues, 110-111, numéro spécial, « De l'anthropologie de l'autre à la reconnaissance d'une autre anthropologie » :337-370. 
Dozon, J.-P., 2003 Frères et sujets. La France et l'Afrique en perspective, Paris, Flammarion. GLUCKMAN, M., 1940 « Analysis of a Social Situation in Modern Zululand. The Social Organization of Modern Zululand », Bantu Studies, 14 (mars) : 129 ; (juin) : 147-174.

HESS, R., 2005 Gérard Althabe, Une biographie entre ici et ailleurs, Paris, L'Harmattan. IMBERNON, J., 2008 « Des populations réfugiées frontalières et leurs impacts, l'exemple de la Guinée forestière ", Géoconfluences, juin, <http://geoconfluences.ens-lsh.fr/doc/typespace/frontier/FrontDoc3.htm>. KLEIN, N., 2008 La stratégie du choc, Arles, Actes Sud. KouvouAma, A. ET AL. (dir.), 2007 Figures croisées d'intellectuels. Trajectoires, modes d'action, productions, Paris, Karthala.

LAPIERRE, N., 2004 Pensons ailleurs, Paris, Stock.

L’HOMME, 2008 Numéro spécial, « Miroirs transatlantiques. Circulation des savoirs et mal entendus féconds entre les États-Unis et l'Europe, y compris la France », 187- 188.

MABANCKOU, A., 2009 L'Europe depuis l'Afrique, Paris, Naïve.

MBEMBE, A., 2005a [2000] De la postcolonie. Essai sur l'imagination politique dans l'Afrique contemporaine, Paris, Karthala.

—, 2005b « La république désœuvrée : La France à l'ère post-coloniale », Le Débat, 137 : 159-175.

MESNARD, P., 2002 La Victime écran. La représentation humanitaire en question, Paris, Textuel.

MIGREUROP, 2009 Atlas des migrants en Europe. Géographie critique des politiques migratoires (sous la direction d'o. Clochard), Paris, Armand Colin.

MITCHELL, J.-C., 1956 The Kalela Dance, Aspects of Social Relationships among Urban Africans in Northern Rhodesia, Manchester, Manchester University Press (trad. française et prés. de M. Agier et S. Nahrath, Enquête, 4, 1997 : 213-243).

PANDOLFI, M., 2000 « Une souveraineté mouvante et supracoloniale », Multitudes, 3 : 97-105.

RANCIÈRE, J., 1995 La Mésentente. Philosophie et politique, Paris, Galilée.

RUFFIN, J.-C., 1994 L’Aventure humanitaire, Paris, Gallimard (« Découvertes »).

SMOUTS, M.-C. (dir.), 2007 La Situation postcoloniale. Les Postcolonial Studies dans le débat français, Paris, Presses de Sciences Po.

STOLER, A. L., 2008 « Imperial Debris : Reflections on Ruins and Ruination », Cultural Anthropology, $23(2): 191-219$.

THOLONIAT, Y. \& de L’ESTOILE, B., 2008 Présentation de la traduction française de l'article de M. Gluckman, op. cit., Genèses, 72 (3) : 119-155.

TROUBÉ, C., 2009 « La fin de l'humanitaire sans frontières », Grotius, juin 2009,

$<$ http://www.grotius.fr/node/308>.

VALlAEYS, A., 2004 Médecins Sans Frontières. La biographie, Paris, Fayard.

VOLLAIRE, C., 2007 Humanitaire, le cœur de la guerre, Paris, L'insulaire. 


\section{NOTES}

1. ERM : Enfants réfugiés du Monde; $H C R$ : Haut commissariat des Nations Unies pour les réfugiés ; MSF : Médecins sans frontières ; ACF : Action contre la faim ; PU : Première urgence.

2. Malgré son titre, La situation postcoloniale. Les Postcolonial Studies dans le débat français, ce n'est pas tant la question de l'analyse de la «situation " (coloniale ou postcoloniale) qui est étudiée dans cet ouvrage, mais la prise en compte d'un passé colonial plus ou moins présent dans les anciennes métropoles, tel qu'il est perçu et interprété en France depuis le milieu du XXe siècle. Le bilan est riche et utile, à l'image de l'introduction de Marie-Claude smouts (2007: 25-66). Il laisse cependant en chantier la question de l'analyse concrète des situations postcoloniales en général et aussi, bien sûr, sur les lieux mêmes de la colonisation passée.

3. On s'explique ainsi le titre français donné à l'ouvrage, de fait plus "global» que "postcolonial» d'Arjun APPADURAÏ (1996), Modernity at Large. Cultural Dimensions of Globalization, devenu en français Après le colonialisme. Les conséquences culturelles de la globalisation. À l'inverse, Dipesh CHAKRABARTY (2000) dont l'ouvrage appelle à la «provincialisation » de l'Europe dans la pensée postcoloniale, reconnaît lui-même que son essai est "un produit de la mondialisation». C'est aussi, peut-on ajouter, un produit de l'exil dans un monde mondialisé, l'auteur déclarant que c'est après avoir quitté l'Inde que la question s'est imposée à lui de définir ce qu'on peut appeler un relativisme local des théories du monde (CHAKRABARTY 2009: 28). La question est d'autant plus évidente quand on est soi-même dans l'exercice d'une pensée déplacée, laquelle à son tour pose la question de sa propre objectivation et réflexivité (LAPIERRE 2004); voir également les réflexions de Zygmunt BAUMAN (2010) qui, dans un entretien avec Benedetto Vecchi, évoque son propre exil et sa propre désidentification nationale comme épreuve cruciale pour penser l'arbitraire des frontières et des identités.

4. Une irritation et quelques raccourcis polémiques que partagent, de manière assez surprenante, Jean-François BAYART (2010) et Jean-Loup AMSELLE (2008) provisoirement réunis pour ce qui ressemble plutôt à un baroud d'honneur... même si c'est à partir d'analyses différentes des contextes et fondements du courant postcolonial, ethnicistes pour Jean-Loup Amselle, dépolitisés et déshistoricisés pour Jean-François Bayart.

5. Dipesh CHAKRABARTY (2009: 53) croit devoir se défendre sur ce point en expliquant que son besoin de "provincialiser l'Europe " prend sa source dans le marxisme et le post-structuralisme européens eux-mêmes, et qu'il ne vise pas un rejet de la pensée européenne.

6. On peut entendre dans ce contexte le rejet de l'anthropologie et plus spécifiquement de l'ethnologie "africaniste" par les chercheurs africains de sciences sociales, politiques et historiques, comme position épistémologique et non personnelle, bien entendu, puisqu'il n'empêche ni les collaborations individuelles ni la pratique anthropologique des chercheurs africains. Ce « combat » remet clairement en question la construction des objets anthropologiques et le nécessaire décentrement de l'anthropologie elle-même dans le monde présent; voir Jean copans (2007). Pour une mise en perspective de ces débats, voir le compte- rendu des débats organisés à l'EHESS par le Centre d'études africaines, «Les épistémès africaines dans le monde », 21-22 juin 2007, <http://ceaf.ehess.fr/ document.php?id=523>. 
7. Voir Y. tholoniat \& B. de l'estoile (2008) pour la présentation de son article.

8. À propos de l'anthropologie situationnelle, je me permets de renvoyer à M. AGIER (2009).

9. Dans ce sens, on notera que c'est pour une large part dans l'étude des campements noirs de Brazzaville (Poto Poto et Bacongo) et de leurs écarts d'avec la ville blanche, que Balandier peut découvrir ce qui constitue la situation coloniale, ce qui ne signifie pas que le tout de l'Afrique vécue à ce moment-là soit une " société coloniale ». Dans la présentation de la réédition (en 1985) de l'ouvrage initialement publié la même année que Sociologie actuelle de l'Afrique noire (1955), G. BALANDIER note: "L'espace de l'agglomération brazzavilloise devenait ainsi un lieu où s'effectuait l'inscription matérielle d'une "situation coloniale" ", en particulier dans l'opposition entre la ville blanche officielle et les nouveaux faubourgs de migrants et travailleurs africains.

10. Notons d'ailleurs que l'empathie méthodologique de l'anthropologie d'Althabe prolonge une position assumée dans une trajectoire à la fois personnelle et politique, qu'elle soit celle de son propre parcours à partir d'un monde socialement "subalterne » (HESS 2005), ou celle de l'engagement politique d'un chercheur, non pas abstrait mais en pratique, qui lui a notamment valu son expulsion de l'ORSTOM (actuel IRD) au début des années 1970, après sa participation au mouvement révolutionnaire malgache des années 1960. De même, l'édition originale des Fleurs du Congo, en 1972, est une longue mise en perspective par Althabe d'un texte révolutionnaire diffusé par le mouvement lumumbiste et qui donna son titre à l'ouvrage, le texte d'Althabe étant dans la première édition, un « commentaire » de ce manifeste.

11. Sur les différents registres (intellectuels, politiques, sociaux) de relations ayant constitué un désir et un besoin réciproques de l'autre chez les Africains et les Français dans la période coloniale et indépendantiste, voir Jean-Pierre Dozon (2003). Pour leurs prolongements actuels, voir Abel KoUvoUAMA ET AL. (2007) et, plus largement, les travaux du Réseau acteurs émergents créé en 2000 (Fondation de la Maison des sciences de l'Homme).

12. On peut ainsi lire, sous l'angle de cette historicité postcoloniale en forme d'appropriation et transformation africaines de la France, l'histoire de la francophonie africaine qui ne se confond pas avec une francophilie politique (MBEMBE 2005a ; BAYART $2010: 35)$.

13. Voir des informations et réflexions plus développées sur la vie quotidienne dans les camps de réfugiés et le mouvement humanitaire dans AGIER (2008).

14. On peut en faire remonter l'origine aux «Dames de la charité » créées par saint Vincent de Paul en 1617, ainsi que, à leur suite, aux Confréries de la charité et aux maisons et hospices pour indigents, enfants des rues, réfugiés et vagabonds, qui connurent un succès considérable en France dans la première moitié du XVII ${ }^{e}$ siècle, ainsi qu'un usage politique inespéré quand le pouvoir monarchique en fit un moyen de contrôle et de mise à l'écart « des pauvres, des malades, de tous ceux auxquels la misère confère une paradoxale et dangereuse liberté » note Jean-Christophe RUFFIN (1994: 26).

15. À propos de l'image de la "victime absolue » et son effet de dépolitisation, voir Jacques RANCIÈRE (1995: 169-188). 
16. Anne VALLAEYS (2004) relate en détail la naissance et l'histoire de MSF, en particulier la rencontre entre «Biafrais » et «Tonusiens » qui aboutit à la création de l'ONG dans les locaux de l'hebdomadaire.

17. UNOCHA : Bureau de l'ONU pour la coordination des affaires humanitaires.

18. ECHO : Bureau de l'Union européenne pour l'aide humanitaire.

19. Principal parti de droite en France, d'abord Union pour la majorité présidentielle, puis Union pour un mouvement populaire.

20. Organisation du Traité de l'Atlantique Nord.

21. Une autre solution est la privatisation rampante, aussi bien du hard power que du soft power... ainsi la présence en Irak, en Somalie, au Yemen, et en Afghanistan (autant que dans la post-catastrophe de la Nouvelle Orléans) des mercenaires de l'entreprise Blackwater oblige les organisations humanitaires à s'interroger en pratique et en théorie sur la réduction et l'insécurité croissante de ce qu'il était convenu d'appeler depuis les années 1980 «l'espace humanitaire» et sur leurs liens avec les forces armées...

22. Voir Christian troubÉ (2009), ainsi que le débat qui a suivi sur le même site.

23. De son côté, Christiane vollaire (2007: 66-83) analyse, exemples à l'appui, les standards contemporains de "l'esthétique humanitaire» et les fonctions désocialisantes des images produites et diffusées pour scandaliser et émouvoir.

24. Voir Rony BRAUMAN, «Arche de Zoé, quelles responsabilités?» CRASH-MSF, 2008, $<$ http://www.msf-crash.org/drive/9719-rb-2008-arche-de-zoe-quelles-responsabilitesfr-transcript-p.5.pdf>.

25. La forme du camp est le parangon de ce gouvernement humanitaire, mais il se reproduit, avec son action et sa puissance "souveraines", dans toute situation évidemment localisée.

26. «UN» (United Nations) est le mot qui désigne le plus souvent l'ensemble indifférencié des acteurs de l'intervention internationale humanitaire.

\section{RÉSUMÉS}

Résumé

Cet article examine le contexte de l'intervention humanitaire en Afrique ces trente dernières années. Instaurant un nouveau type de relation, compassionnelle, suspicieuse et victimaire, entre Blancs et Noirs, l'intervention humanitaire crée dans le même temps ses propres espaces et situations caractérisés par l'exception, l'extraterritorialité et la relégation. L'analyse de cette présence de l'humanitaire en Afrique passe nécessairement par une interrogation sur la généalogie postcoloniale où elle se situe et qui lui donne, en partie au moins, son sens, alors même que le récit humanitaire se construit comme un des récits majeurs de la mondialisation. Alors que sa puissance se confirme comme idéologie et gouvernement des indésirables, le mouvement humanitaire traverse une crise face à laquelle certains prônent sa « désoccidentalisation », faisant écho à la polémique postcoloniale dans les sciences sociales. 
Abstract

This paper examines the background to humanitarian intervention over the past thirty years. By establishing a new type of relationship between Whites and Blacks-one of compassion, suspicion and victimhood-humanitarian intervention has created its own spaces and situations, marked by exception, extraterritoriality and relegation. To analyse the humanitarian presence in Africa it is necessary to understand its postcolonial genealogy, which, to some extent, gives meaning to it, even as the humanitarian narrative is being constructed as one of the major narratives of globalisation. While its power lies in the ideology and governance of "undesirables", the humanitarian movement is currently in crisis, leading some people to call for its "deWesternization", echoing the current postcolonial controversy in the social sciences.

INDEX

Mots-clés : Guinée, analyse situationnelle, camps de réfugiés, intervention humanitaire, postcolonialisme, sciences sociales

Keywords : Guinea, Situational Analysis, Refugee Camps, Humanitarian Intervention, Postcolonialism, Social Sciences

\section{AUTEUR}

\section{MICHEL AGIER}

Institut de recherche pour le développement (IRD) ; École des hautes études en sciences sociales (EHESS), Paris. 